Tropical Journal of Pharmaceutical Research February 2014; 13 (2): 303-305

ISSN: $1596-5996$ (print); 1596-9827 (electronic) (C) Pharmacotherapy Group, Faculty of Pharmacy, University of Benin, Benin City, 300001 Nigeria.

All rights reserved.

\title{
New-Onset Psychosis in a Multi-Drug Resistant Tuberculosis Patient on Cycloserine in Calabar, Nigeria: A Case Report
}

\author{
Akaninyene A Otu ${ }^{1}$, Jonah B Offor ${ }^{2}$, Ibene A Ekpor ${ }^{1}$ and Oladimeji Olarenwaju ${ }^{3}$ \\ ${ }^{1}$ University of Calabar Teaching Hospital, '2Lawrence Henshaw Memorial Hospital, ${ }^{1}$ University of Calabar Teaching Hospital, \\ Calabar, ${ }^{3}$ Damien Foundation, Ibadan, Nigeria.
}

*For correspondence: Email: akanotu@yahoo.com; Tel: +234-8105723133.

\begin{abstract}
Drug-resistant tuberculosis poses a serious challenge to global control of TB. These forms of TB do not respond to the standard six-month treatment; it can take two years or more to treat with category IV drugs that are less potent, more toxic and much more expensive. Treatment of multi-drug resistant tuberculosis is still evolving in Nigeria. This case report highlights the side effects of cycloserine used to treat a multi-drug resistant tuberculosis patient in Calabar. Five days into therapy, he became disoriented, abusive and physically aggressive. He also displayed negativisim with paranoid delusions and insomnia. He was managed by a psychiatrist with anti-psychotic drugs. The dose of cycloserine was also reduced while that of pyridoxine was increased. He remained in a state of periodic confusion and psychosis for nine days after which his condition ameliorated. It is imperative that clinicians involved in treating multi-drug resistant tuberculosis are conversant with the side effects of category IV drugs. Acute psychosis from cycloserine toxicity requires prompt intervention by trained medical personnel using the relevant psychotropic medications. Reduction in dosage or outright stoppage of cycloserine in such situations should be considered. Use of pyridoxine in large doses also appears to be beneficial.
\end{abstract}

Keywords: Psychosis, Tuberculosis, Cycloserine, Multidrug resistance, Category IV drugs

Tropical Journal of Pharmaceutical Research is indexed by Science Citation Index (SciSearch), Scopus, International Pharmaceutical Abstract, Chemical Abstracts, Embase, Index Copernicus, EBSCO, African Index Medicus, JournalSeek, Journal Citation Reports/Science Edition, Directory of Open Access Journals (DOAJ), African Journal Online, Bioline International, Open-J-Gate and Pharmacy Abstracts

\section{INTRODUCTION}

This is a case report of a 28 year old male pulmonary tuberculosis patient presenting at the Lawrence Henshaw Memorial Hospital Calabar, Nigeria for care. Drug susceptibility testing done on his sputum sample showed resistance to isoniazid, rifampicin, ethambutol and streptomycin.

He had been previously diagnosed with tuberculosis in 2007 for which he received treatment for just three months. This three months of therapy consisted of 2 months of isoniazid, rifampicin, ethambutol and pyrazinamide and 1 month of isonizid and rifampicin. He then decided to discontinue treatment because he felt much better. In 2009, he was re-treated for tuberculosis for 6 months during which streptomycin injection was incorporated.

He was not on any long-term medications and had no background mental disorder.

$\mathrm{He}$ was unmarried, did not smoke and did not abuse illegal drugs or alcohol.

Examination revealed a young man who was pale with no peripheral lymph node enlargement 
or finger clubbing. His weight was $55 \mathrm{~kg}$, height $1.65 \mathrm{~m}$ and Body Mass Index (BMI) 20.2. His vital signs were temperature $36.8^{\circ} \mathrm{C}$, respiratory rate $22 / \mathrm{min}$, pulse $90 / \mathrm{min}$ and blood pressure $120 / 80 \mathrm{mmHg}$.

Chest examination revealed coarse crackles in the left middle and lower lung zones.

His chest $x$-ray showed fibrotic strands in the left upper lung zone with diffuse pulmonary infiltrates in the left middle and lower lung zones.

His haemoglobin estimation was $11.6 \mathrm{~g} / \mathrm{dl}$ and the white cell count with differentials were normal. Pure tone audiometry revealed a bilateral mild conductive hearing loss. His urinalysis, serum electrolytes, liver function, thyroid function and fasting blood sugar tests were all normal. He also tested negative for human immunodeficiency virus.

A diagnosis of multidrug resistant (MDR) pulmonary tuberculosis was made and he was admitted into the MDR-TB ward of the facility. He was commenced on a supervised Category IV treatment regimen. The 8-month intensive phase of this regimen comprised of daily doses of pyrazinamide $1.5 \mathrm{~g}$, kanamycin $1 \mathrm{~g}$, levofloxacin $750 \mathrm{mg}$, protionamide $750 \mathrm{mg}$, cycloserine $750 \mathrm{mg}$ and pyridoxine $150 \mathrm{mg}$.

Three days after commencement of the above regimen, he was noticed to be soliloquizing for about half an hour at night. When asked about this the next morning, he said he had a vivid dream in which his deceased grandmother appeared and had a conversation with him. He appeared otherwise normal and his medications were continued.

Five days into therapy, he became disoriented, abusive and physically aggressive. $\mathrm{He}$ also displayed negativism with paranoid delusions and insomnia. A diagnosis of acute psychosis (probably from cycloserine use) was entertained. However, his serum level of cycloserine could not be assessed due to lack of facilities. He had to be physically restrained and received a dose of intravenous diazepam. He was then started on a psychiatric regimen of chlorpromazine, haloperidol and artane. This sedated him somewhat but he continued to be abusive to the staff and requested to be discharged. His appetite became very poor. He developed torticollis $24 \mathrm{~h}$ later and the dose of artane was increased. On the $7^{\text {th }}$ day of therapy, he tried to escape from the facility by scaling the perimeter fence. He was discovered in the process and restrained. His anti-tuberculosis medications were then reviewed. The dose of cycloserine was reduced from $750 \mathrm{mg}$ once daily to $500 \mathrm{mg}$ daily. This dose of $500 \mathrm{mg}$ was then delivered as $250 \mathrm{mg}$ twice daily instead of a single daily dose. The dose of pyridoxine was also increased to $200 \mathrm{mg}$ daily. He was also put under close observation. A re-check of his liver function and electrolytes was done to exclude liver or renal damage.

He remained in this state of periodic confusion and psychosis for 9 days after which his condition ameliorated. He gradually became less confrontational and more oriented till he regained his pre-treatment mental state. Olanzapine was introduced and chlorpromazine was tailed off. $\mathrm{He}$ continued on his second line anti-tuberculosis drugs with no further incident.

\section{DISCUSSION}

Drug resistant TB poses a serious challenge to global control of TB [1]. Resistance to one antiTB drugs is known as mono-resistance. Multidrug resistant TB (MDR-TB) is resistance to at least isoniazid and rifampicin, the two key first line anti-TB drugs in short course chemotherapy [1]. These forms of TB do not respond to the standard six-month treatment with anti-TB drugs and can take two years or more to treat with drugs that are less potent, more toxic and much more expensive [2].

Nigeria has the tenth largest burden of TB cases in the world. The 2011 World Health Organization (WHO) report states that Nigeria has an estimated MDR-TB rate of 2.2 and $9.4 \%$ among new and re-treatment TB cases, respectively. Nigeria is therefore ranked 15th among the 27 High Burden Countries for MDRTB [3].

To the best of our knowledge, the commencement of Category IV treatment for the index patient is a landmark achievement in the annals of MDR-TB control in Nigeria. This patient was the very first to be admitted to the only MDR-TB treatment facility in the South-South zone of Nigeria which is comprised of six states in the federation. Thus, the acute psychosis recorded in him was a cause of great concern for the national TB control programme. It is noteworthy that the Psychiatrist on the Clinical Expertise Committee of the MDR-TB facility was instrumental to the success recorded in the management of this crisis.

The most likely diagnosis to explain this patient's psychosis was cycloserine toxicity. The recorded neuropsychiatric side effects of cycloserine include psychosis, depression, aggression, 
paranoia, anxiety, confusion and suicidal ideation [4]. Cycloserine has a narrow therapeutic window and neurotoxicity from its use is common as it readily crosses the blood brain barrier and decreases gamma amino butyric acid (GABA) production [5]. Ideally, patients receiving more than $500 \mathrm{mg}$ daily and those with renal dysfunction or suspected toxicity should have their Cycloserine plasma concentrations monitored and kept below $30 \mathrm{mg} / \mathrm{ml}$. Concurrent use of alcohol with cycloserine increases the risk of developing psychosis and seizures [4]. Severe Cycloserine neurotoxicity can be managed by suspending the drug for a while. It can then be re-introduced at low dosages once the patient is back to normal. Due to the long half life of cycloserine, patients with side effects may take days or weeks to return to normal. For the patients who cannot tolerate the re-introduction of cycloserine, an alternative drug can be utilized [5].

For less severe manifestations of cycloserine toxicity, the dosage of cycloserine can be reduced. Antipsychotics are very useful for managing neuropsychiatric effects and can be continued throughout the duration of MDR-TB treatment [5].

\section{CONCLUSION}

As the treatment of MDR-TB fully comes on stream in Nigeria, it is imperative that clinicians are conversant with the full spectrum of untoward side effects that patients on category IV drugs could manifest with. Acute psychosis occurring in this setting from cycloserine toxicity requires prompt intervention by trained medical personnel using relevant psychotropic medications. It is also expedient to have a psychiatrist on the multidisciplinary team to oversee the management of such neuropsychiatric manifestations. Reduction in dosage or outright stoppage of cycloserine in such situations should be considered. Use of pyridoxine in large doses also appears to be beneficial. In settings where the monitoring of drug serum levels is not possible, Cycloserine toxicity may be prevented by giving the lowest possible dose under close hospital supervision.

\section{CONFLICT OF INTEREST}

The author declare that there is no conflict of interest.

\section{REFERENCES}

1. Rijal KR, Ghimire $P$, Rijal D, Bam DS. The pattern of anti tuberculosis drug resistance in pulmonary tuberculosis patients.J Inst Med 2005; 27: 26-28.

2. World Health Organization. Multidrug and extensively drug resistant TB (M/XDR- TB) 2010 Global Report On Surveillance and Response 2010. Geneva: WHO; 2010; $p 24$

3. World Health Organization. Global tuberculosis control: WHO Report 2011. Geneva: WHO; 2011; $p 34$.

4. United States Agency for International Development. New onset psychosis. TB Care II Online 2012. [cited 2012 Jan 21]. Available from: http://tbcare.gotpantheon.com/comment/20

5. Coyne KM, Pozniak AL, Lamorde $M$, Boffito $M$. Pharmacology of second-line antituberculosis drugs and potential for interactions with antiretroviral agents. AIDS 2009; 23: 437-446. 